\title{
A Proposed SM-IMSAF Algorithm with Fast Convergence Rate
}

\author{
Long Shi, Haiquan Zhao* \\ School of Electrical Engineering, Southwest Jiaotong University, Chengdu City, Sichuan Province, China. \\ * Corresponding author. Tel: 13550321407; email: hqzhao@home.swjtu.edu.cn \\ Manuscript submitted June 5, 2015; accepted May 8, 2016. \\ doi: 10.17706/ijcce.2017.6.1.57-66
}

\begin{abstract}
In order to obtain a fast convergence rate, we propose a novel algorithm at the basis of the improved multiband-structured subband adaptive filter algorithm (IMSAF). The proposed algorithm incorporates the idea of set-membership into the IMSAF (SM-IMSAF). The update equation of the proposed SM-IMSAF is derived by using the Lagrange Multiplier method. Due to the effect of set-membership, the proposed SM-IMSAF achieves a better performance than some existing well-known algorithms. The simulation experiments are carried out under the condition of the system identification applications. Considering the practical condition, exact-modeling as well as under-modeling is taken into account in the simulations. At the same time, the tracking ability of SM-IMSAF algorithm is also researched when the unknown system mutates. The simulation results verify the superiority of the SM-IMSAF algorithm.
\end{abstract}

Key words: SM-IMSAF, system identification, set-membership, exact-modeling, tracking ability.

\section{Introduction}

The adaptive filtering plays an important role in the signal processing applications, such as system identification, active noise control, acoustic echo cancellation and distributed networks [1]-[5].

The original normalized least-mean-square (NLMS) algorithm performs well when the input signal is Gaussian white signal. However, its convergence rate becomes slow when the input signal is colored signal [6]. In order to speed up the convergence rate, the multiband-structured subband adaptive filter algorithm (MSAF) was proposed in [7]. The MSAF algorithm achieves good convergence performance when the input signal is colored signal. Moreover, the convergence rate becomes faster with the increasing of subband number. However, the MSAF algorithm uses a fixed step size, leading to this algorithm sacrifices the steady-state error for a fast convergence rate. In order to overcome this drawback, [8] proposed a set-membership subband adaptive filter algorithm (SM-SAF). The SM-SAF algorithm guarantees a fast convergence rate as well as a quite low steady-state error. Of course, the affine projection algorithm (APA) [9] is also widely researched because of its advantage that improves the convergence rate in dealing with the colored signal. A set-membership affine projection algorithm (SM-APA) was proposed in [10]. This algorithm has a faster convergence rate compared with the common APA algorithm. At the same time, its steady-state error is lower than common APA algorithm. To further improve the performance of the SM-APA, several variants based on different strategies have been proposed [11]-[14].

Considering that both MSAF and APA perform well in terms of the convergence rate when the input signal is colored, Yang proposed an improved multiband-structured subband adaptive filter (IMSAF) algorithm 
[15], which combines the subband adaptive filter with affine projection method. The IMSAF algorithm achieves faster convergence than the MSAF algorithm and APA algorithm.

In this paper, we propose a new algorithm, where the idea of set-membership is added in the IMSAF algorithm (SM-IMSAF).

This paper will be organized as follows. Section 2 introduces the IMSAF algorithm. Section 3 clarifies the proposed SM-IMSAF algorithm. Section 4 presents the simulation experiments including the comparison of different algorithms and analyses of the update rate of the tap-weight vector equation. Section 5 reveals our conclusions.

\section{Review of the IMSAF Algorithm}

In the view of the fact that the IMSAF algorithm was proposed on the basis of the multiband-structured subband adaptive filter (MSAF) algorithm [7], we have necessity to introduce the MSAF algorithm.

The structure of the MSAF is shown in Fig. 1. The desired response is achieved by the following model

$$
d(n)=\mathbf{w}_{o}^{T} \mathbf{u}(n)+\eta(n)
$$

where $\mathbf{u}(n)=[u(n), u(n-1), \cdots, u(n-L+1)]^{T}$ is the input vector, the superscript $T$ denotes transposition of a vector or matrix, $\mathbf{w}_{o}=\left[w_{0}, w_{1}, \cdots, w_{L-1}\right]^{T}$ denotes the tap-weight vector of the unknown system, $\eta(n)$ represents the measurement noise, and $L$ is the length of tap-weight vector.

As is shown in Fig. 1, the desired signal $d(n)$ and the input signal $u(n)$ are respectively partitioned into $N$ subband signals $d_{i}(n), u_{i}(n)$ by means of analysis filters $H_{i}(z)$, where $i=0, \cdots, N-1$. The output signal $y_{i}(n)$ is obtained by filtering from $u_{i}(n)$ using the adaptive filter. The notation $\downarrow N$ represents $N$-fold decimation and $\uparrow N$ represents $N$-fold interpolation.

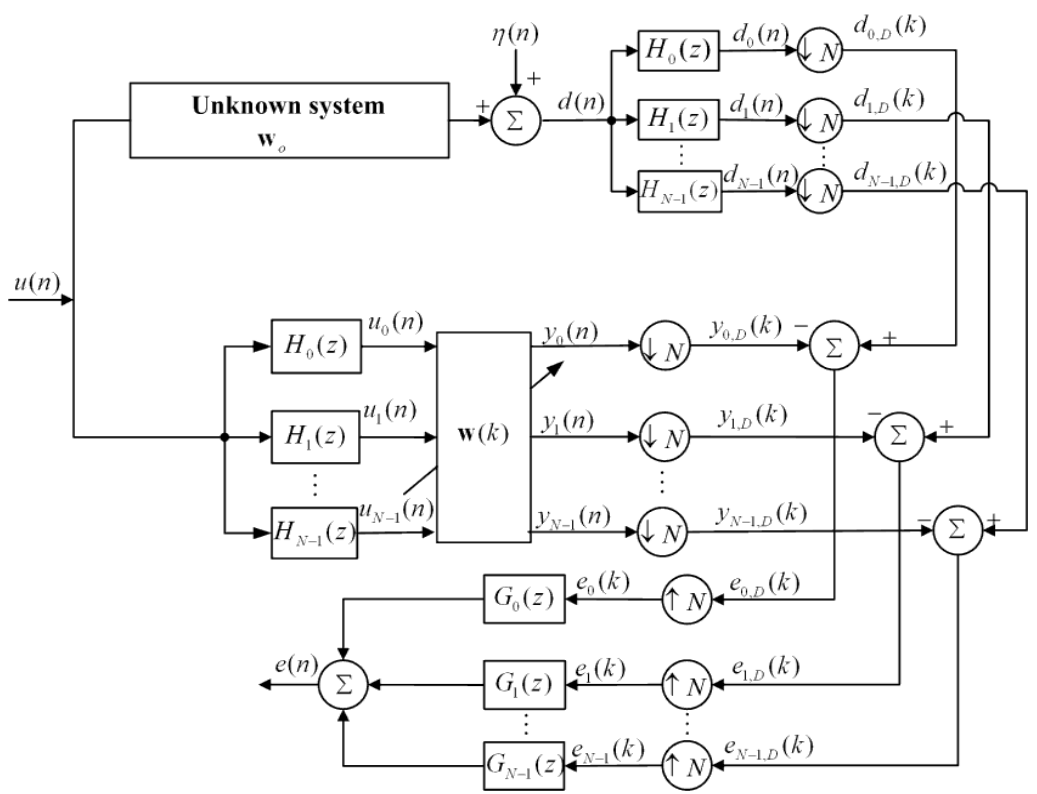

Fig. 1. Block diagram of multiband-structured subband adaptive filter.

The signals $d_{i, D}(k)$ are generated by decimating from the subband signals $d_{i}(n)$ with the help of $N$-fold decimation. The principle of generating $y_{i, D}(k)$ is the same as generating $d_{i, D}(k)$. Then, a priori decimated subband error is defined as 


$$
e_{i, D}(k)=d_{i, D}(k)-\mathbf{w}^{T}(k) \mathbf{u}_{i}(k) \quad \text { for } i=0, \cdots, N-1
$$

and a posteriori decimated subband error is defined as

$$
\xi_{i, D}(k)=d_{i, D}(k)-\mathbf{w}^{T}(k+1) \mathbf{u}_{i}(k) \quad \text { for } i=0, \cdots, N-1
$$

where $\mathbf{u}_{i}(k)=\left[u_{i}(k N), \cdots, u_{i}(k N-M+1)\right]^{T}$ denotes the regression vector of the $i$ th subband signal and $\mathbf{w}(k)=\left[w_{0}(k), \cdots, w_{M-1}(k)\right]^{T}$ is the tap-weight vector of the adaptive filter. $M$ is the length of the adaptive filter. Motivated by the affine projection algorithm, the IMSAF algorithm is obtained by adding the idea of APA into the MSAF algorithm. The derivation of IMSAF algorithm is based on the principle of minimal distance by canceling the most $P$ a posteriori errors in each of the $N$ subbands, i.e. a total of $P * N$ error signals at each iteration $k$

$$
\min _{\mathbf{w}(k+1)}\|\mathbf{w}(k+1)-\mathbf{w}(k)\|^{2} \quad \text { subject to } \xi_{D}(k)=\mathbf{0}
$$

where $\mathbf{0}$ is a $N P \times 1$ null matrix, $\xi_{D}(k)$ is defined as

$$
\begin{aligned}
& \xi_{D}(k)=\left[\xi_{0, D}(k), \cdots, \xi_{0, D}(k-P+1),\right. \\
& \\
& \xi_{1, D}(k), \cdots, \xi_{1, D}(k-P+1), \\
&\left.\cdots, \xi_{N-1, D}(k), \cdots, \xi_{N-1, D}(k-P+1)\right]^{T} \\
&= \mathbf{d}_{D}(k)-\mathbf{U}^{T}(k) \mathbf{w}(k+1)
\end{aligned}
$$

The tap-weight vector $\mathbf{w}(k)$ is updated as follows

$$
\mathbf{w}(k+1)=\mathbf{w}(k)+\mu \mathbf{U}(k)\left[\mathbf{U}^{T}(k) \mathbf{U}(k)+\varepsilon \mathbf{I}\right]^{-1} \mathbf{e}_{D}(k)
$$

where $\mathbf{I}$ is a $N P \times N P$ identity matrix, $\varepsilon$ denotes a regularization parameter, $\mu$ represents the step size. The other relative variables are defined as follows

$$
\begin{aligned}
& \mathbf{U}(k)= {\left[\mathbf{u}_{0}(k), \mathbf{u}_{0}(k-1), \cdots, \mathbf{u}_{0}(k-P+1),\right.} \\
& \mathbf{u}_{1}(k), \mathbf{u}_{1}(k-1), \cdots, \mathbf{u}_{1}(k-P+1), \\
& \cdots,\left.\mathbf{u}_{N-1}(k), \cdots, \mathbf{u}_{N-1}(k-P+1)\right] \\
& \mathbf{d}_{D}(k)= {\left[d_{0, D}(k), \cdots, d_{0, D}(k-P+1),\right.} \\
& d_{1, D}(k), \cdots, d_{1, D}(k-P+1), \\
&\left.\cdots, d_{N-1,0}(k), \cdots, d_{N-1, D}(k-P+1)\right]^{T} \\
& \mathbf{e}_{D}(k)=\left[e_{0, D}(k), \cdots, e_{0, D}(k-P+1),\right. \\
& \mathrm{e}_{1, D}(k), \cdots, e_{1, D}(k-P+1), \\
& \cdots,\left.\mathrm{e}_{N-1, D}(k), \cdots, e_{N-1, D}(k-P+1)\right]^{T} \\
&= \mathbf{d}_{D}(k)-\mathbf{U}^{T}(k) \mathbf{w}(k)
\end{aligned}
$$




$$
\begin{aligned}
\boldsymbol{\eta}_{D}(k)= & {\left[\eta_{0, D}(k), \cdots, \eta_{0, D}(k-P+1),\right.} \\
& \eta_{1, D}(k), \cdots, \eta_{1, D}(k-P+1), \\
& \left.\cdots, \eta_{N-1, D}(k), \cdots, \eta_{N-1, D}(k-P+1)\right]^{T} \\
& =\mathbf{d}_{D}(k)-\mathbf{U}^{T}(k) \mathbf{w}_{o}
\end{aligned}
$$

\section{Proposed SM-IMSAF Algorithm}

\subsection{Introduction of Set-Membership}

As mentioned earlier, we add the idea of set-membership into the IMSAF algorithm, then the SM-IMSAF algorithm is proposed.

Here, we briefly introduce the knowledge of set-membership [10] applied in the adaptive algorithm. We define the constraint set $H_{k}$ containing all vectors $\mathbf{w}$ with estimation errors upper bounded in magnitude by $\gamma$ at time instant $k$.

$$
H_{k}=\left\{\mathbf{w} \in R^{N}:\left|d(k)-\mathbf{w}^{T} \mathbf{u}(k)\right| \leq \gamma\right\}
$$

where $d(k)$ denotes the desired signal, $\mathbf{u}(k)$ represents the input signal vector, $\gamma$ is a design parameter that can vary with the specific application. Then, the membership set $\psi_{k}$ is defined as

$$
\psi_{k}=\bigcap_{i=1}^{k} H_{i}
$$

The core idea of set-membership is that only when $\mathbf{w}(k) \notin H_{k}, \quad \mathbf{w}(k+1)$ will be updated.

\subsection{Derivation of the SM-IMSAF Algorithm}

Some quantities used in the following formulas have been defined in Section 2. Besides, we also need to define that

$$
\begin{gathered}
d_{i, D}(k-j+1)-\mathbf{w}^{T} \mathbf{u}_{i}(k-j+1)=g_{i}(k-j+1) \quad \text { for } i=0, \cdots, N-1 \quad j=1, \cdots, P \\
\mathbf{g}(k)=\left[g_{0}(k), \cdots, g_{0}(k-P+1),\right. \\
g_{1}(k), \cdots, g_{1}(k-P+1), \\
\left.\cdots, g_{N-1}(k), \cdots, g_{N-1}(k-P+1)\right]^{T}
\end{gathered}
$$

We seek $\mathbf{w}(k+1)$ by solving the constrained optimization criterion

$$
\min _{\mathbf{w}(k+1)}\|\mathbf{w}(k+1)-\mathbf{w}(k)\|^{2} \quad \text { subject to } \xi_{D}(k)=\mathbf{g}(k)
$$

then we could solve a constraint minimization problem by constructing the following cost function

$$
J[\mathbf{w}(k+1)]=\frac{1}{2}\|\mathbf{w}(k+1)-\mathbf{w}(k)\|^{2}+\lambda^{T}\left[\xi_{D}(k)-\mathbf{g}(k)\right]
$$

where $\lambda=\left[\lambda_{0}, \lambda_{1}, \cdots, \lambda_{N P-1}\right]^{T}$ is the Lagrange multipliers vector.

Setting the gradient of $J[\mathbf{w}(k+1)]$ with respect to $\mathbf{w}(k+1)$ equal to zero, we get 


$$
\mathbf{w}(k+1)=\mathbf{w}(k)+\mathbf{U}(k) \lambda
$$

Invoking the constraints in (15), we obtain

$$
\begin{aligned}
\mathbf{U}^{T}(k) \mathbf{U}(k) \lambda= & \mathbf{U}^{T}(k) \mathbf{w}(k+1)-\mathbf{U}^{T}(k) \mathbf{w}(k) \\
= & \mathbf{d}_{D}(k)-\mathbf{g}(k)-\mathbf{U}^{T}(k) \mathbf{w}(k) \\
& =\mathbf{e}_{D}(k)-\mathbf{g}(k)
\end{aligned}
$$

The update equation of the tap-weight vector can be obtained by combining (17) with (18)

$$
\mathbf{w}(k+1)=\left\{\begin{array}{lr}
\mathbf{w}(k)+\mathbf{U}(k)\left[\mathbf{U}^{T}(k) \mathbf{U}(k)\right]^{-1}\left[\mathbf{e}_{D}(k)-\mathbf{g}(k)\right], \text { if } \exists\left|e_{i, D}\right|>\gamma \\
\mathbf{w}(k) & \text { otherwise }
\end{array}\right.
$$

According to the reference [10], we define that

$$
\begin{gathered}
\mathbf{e}_{i, D}(k)=\left[e_{i, D}(k), \cdots, e_{i, D}(k-P+1)\right]^{T} \\
\mathbf{g}_{i}(k)=\left[g_{i}(k), \cdots, g_{i}(k-P+1)\right]^{T}
\end{gathered}
$$

We choose that $g_{i}(k-j+1)=e_{i, D}(k-j+1)$ for $j=2, \cdots, P$, which cancels all but the first element of $\mathbf{e}_{i, D}(k)-\mathbf{g}_{i}(k)$. Letting $g_{i}(k)=\gamma \operatorname{sign}\left(e_{i, D}(k)\right)$, a solution lying at the nearest boundary of $H_{k}$, we will reduce (18) to the following formula

$$
\mathbf{U}^{T}(k) \mathbf{U}(k) \lambda=\mathbf{Q}(k)
$$

where

$$
\begin{gathered}
\mathbf{Q}(k)=\left[\begin{array}{cc}
\alpha_{0}(k) e_{0, D}(k) & \begin{array}{c}
0 \cdots 0 \\
P-1
\end{array} \\
\alpha_{1}(k) e_{1, D}(k) & \begin{array}{c}
0 \cdots 0 \\
P-1
\end{array}
\end{array}\right. \\
\left.\cdots \alpha_{N-1}(k) e_{N-1, D}(k) \quad \begin{array}{c}
0 \cdots 0 \\
P-1
\end{array}\right]^{T} \\
\alpha_{i}(k)= \begin{cases}1-\frac{\gamma}{\left|e_{i, D}(k)\right|}, & \text { if }\left|e_{i, D}(k)\right|>\gamma \\
0, & \text { otherwise }\end{cases}
\end{gathered}
$$

Finally, we obtain the update equation of the tap-weight vector as

$$
\mathbf{w}(k+1)=\mathbf{w}(k)+\mathbf{U}(k)\left[\mathbf{U}^{T}(k) \mathbf{U}(k)\right]^{-1} \mathbf{Q}(k)
$$

In order to avoid numerical instability, we have necessity to add a regularization parameter $\varepsilon$ to the diagonal elements of $\mathbf{U}^{T}(k) \mathbf{U}(k)$, then we obtain the following equation

$$
\mathbf{w}(k+1)=\mathbf{w}(k)+\mathbf{U}(k)\left[\mathbf{U}^{T}(k) \mathbf{U}(k)+\varepsilon \mathbf{I}\right]^{-1} \mathbf{Q}(k)
$$

Obviously, if $\gamma$ equals to zero, the SM-IMSAF algorithm will be reduced to the IMSAF algorithm, but the 
only difference is that $\mathbf{e}_{D}(k)$ does not use all values.

\section{Simulation Results}

The simulations are carried out in the context of system identification. We consider both the exact-modeling and under-modeling scenarios. The NMSD, defined as $10 \log _{10} E\left[\boldsymbol{\varepsilon}^{T}(k) \boldsymbol{\varepsilon}(k)\right] /\left(\mathbf{w}_{o}{ }^{T} \mathbf{w}_{o}\right)$, is used to evaluated the performance of the proposed algorithm, where $\boldsymbol{\varepsilon}(k)=\mathbf{w}(k)-\mathbf{w}_{o}$. For the under-modeling scenario, the NMSD is calculated by padding the tap-weight vector of the adaptive filter with $L-M$ zeros [16]. The length of the adaptive filter's tap-weight vector $M$ is 512 . We use the measured impulse response of the acoustic echo path as the unknown system whose sampling rate is $8 \mathrm{kHz}$. For the unknown system, $L$ denotes 512 in the exact-modeling scenario. Correspondingly, $L$ represents 1024 in the under-modeling scenario.

An AR(4) signal, generated by passing a zero-mean white Gaussian noise through a fourth-order system $T(z)=1 /\left(1-0.95 z^{-1}-0.19 z^{-2}-0.09 z^{-3}+0.5 z^{-4}\right)$, is used as input signal for the system identification. We add a white Gaussian noise whose SNR is $30 \mathrm{~dB}$ to the desired output signal. We define that $\gamma(j)=\sqrt{j \sigma_{\eta}^{2}}, \varepsilon=0.01$.All simulation experiments are independently run for 50 numbers

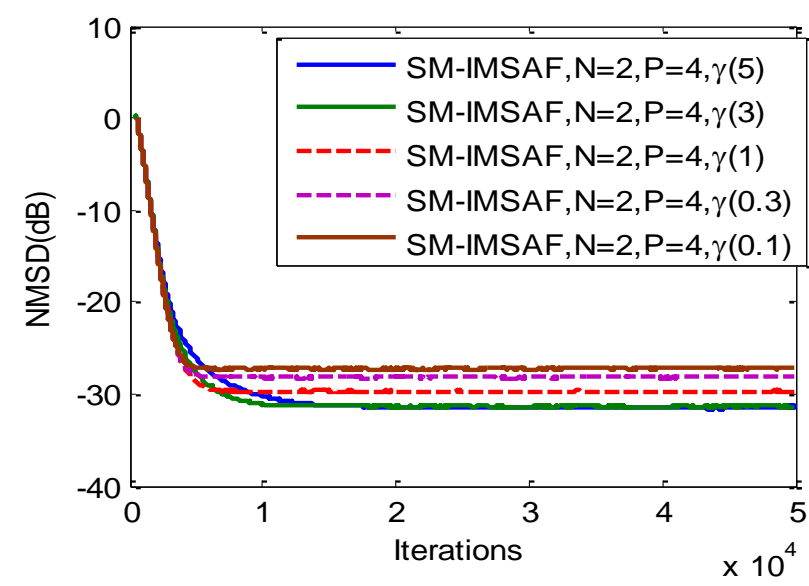

Fig. 2. NMSD curves of the SM-IMSAF algorithm, comparing performance when $\gamma(j)$ takes different values, where $j=5,3,1,0.3,0.1$ for the exact-modeling.

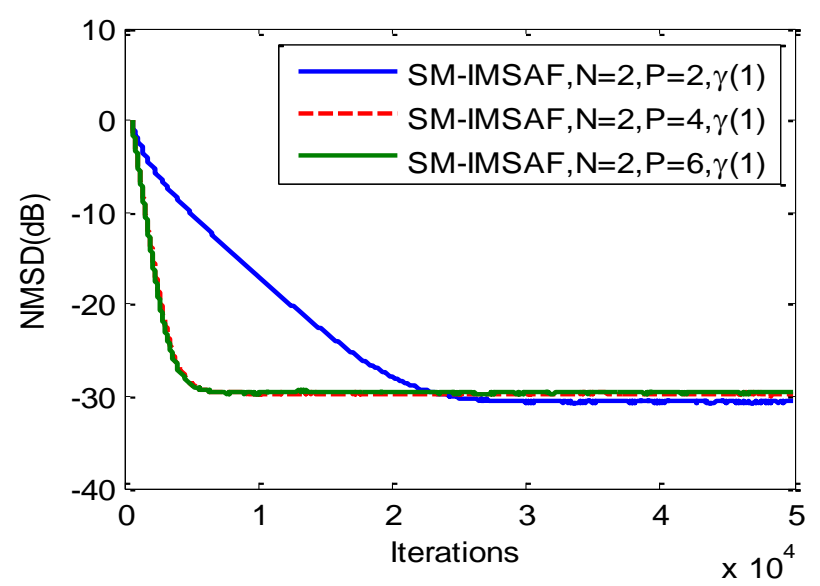

Fig. 3. NMSD curves of the SM-IMSAF algorithm, comparing performance when $P$ takes $2,4,6$ respectively for the exact-modeling. 


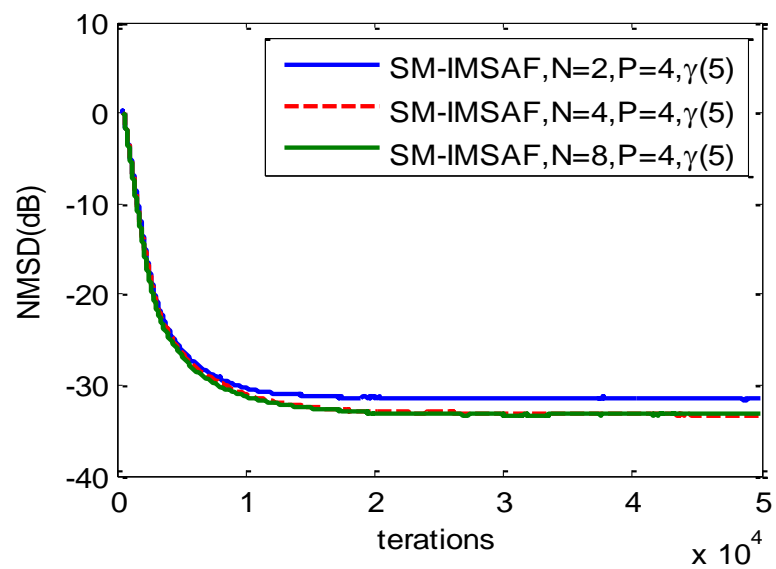

Fig. 4. NMSD curves of the SM-IMSAF algorithm, comparing performance when $N$ takes 2, 4, 8 respectively for the exact-modeling.

\subsection{Comparison of the Simulation Results}

As shown in Fig. 2, the SM-IMSAF algorithm with $\gamma(1)$ has a faster convergence rate than that $\gamma(5)$, $\gamma(3)$, but yields a higher steady misadjustment . Comparing with $\gamma(0.3), \gamma(0.1)$, the SM-IMSAF algorithm with $\gamma(1)$ has a similar convergence rate and a lower steady misadjustment. Fig. 3 demonstrates that there exists no obvious differences in their performance when $P$ takes 4 and 6 respectively. Fig. 4 indicates that the SM-IMSAF algorithm with $N=4$ could achieve a similar performance as that $N=8$.

In order to obtain a quite good performance, we need to make a tradeoff between the convergence rate and steady misadjustment. So, the choice that $N=4, P=4, \gamma(1)$ will be reasonable.

The simulation results from Fig. 5 to Fig. 6 show that the proposed SM-IMSAF algorithm obtain a better performance compared with other three algorithms. For example, the IMSAF algorithm achieve a quite fast convergence rate for $\mu=1$ [15], but compared with the proposed SM-IMSAF algorithm, its steady misadjustment is higher. Even if the unknown system mutates, the proposed SM-IMSAF algorithm still behaves better than other algorithms.

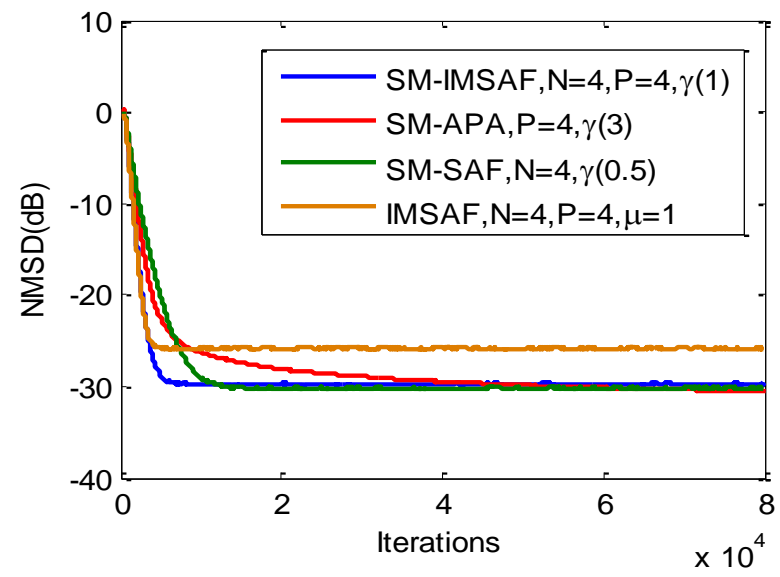

Fig. 5. Comparing SM-IMSAF with SM-APA, SM-SAF, IMSAF for the exact-modeling, where all the algorithms adopt an $\mathrm{AR}(4)$ signal, SNR=30dB.

Taking the under-modeling scenario into account, we obtain results from Fig. 7. The proposed SM-IMSAF algorithm has a faster convergence rate than SM-APA and SM-SAF algorithm when keeping the steady misadjustment approximately equal. Compared with the IMSAF algorithm, the proposed SM-IMSAF 
algorithm achieves a lower steady misadjustment. It is worth noting that when comparing with the SM-IMSAF algorithm, $\gamma(0.01)$ for the SM-SAF algorithm is a quite small value. It means that the tap-weight equation almost updates at each iteration.

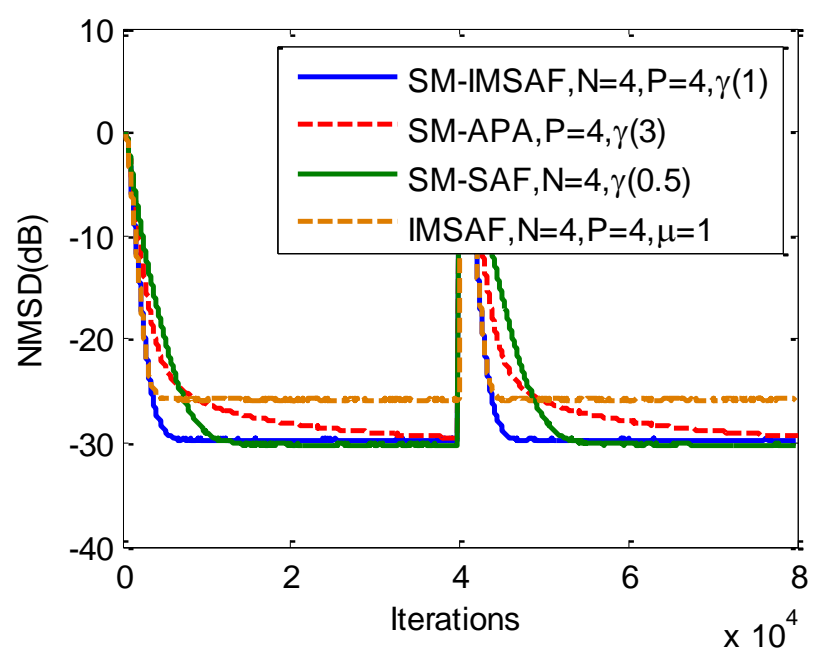

Fig. 6. The unknown system mutates at $4 \times 10^{4}$, comparing the performance of different algorithms for the exact-modeling.

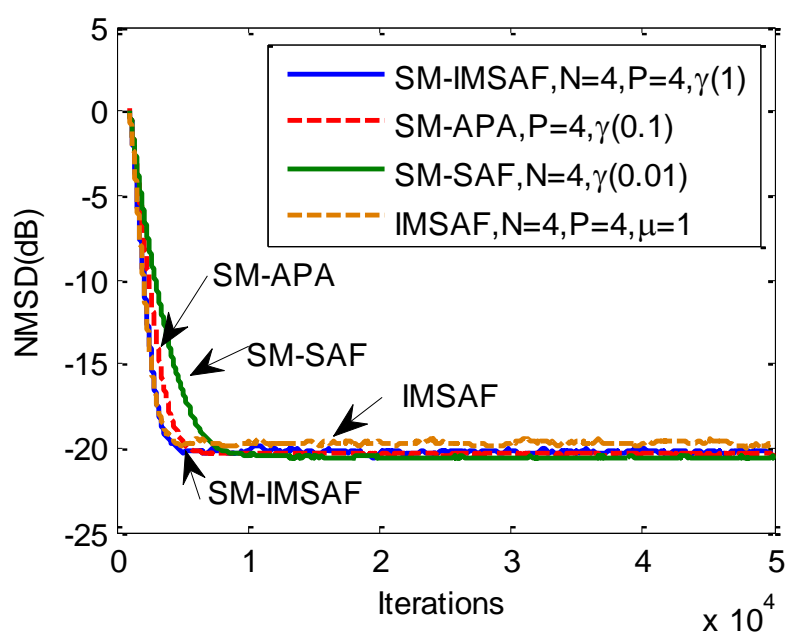

Fig. 7. Comparison of the NMSD curves for different algorithms in the under-model scenario. AR(4) input signal, $\mathrm{SNR}=30 \mathrm{~dB}$.

\subsection{Update Rate of the Tap-Weight Vector Equation}

In general, those algorithms that involve the set-membership will discuss the update rate of tap-weight vector equation [17], a proportion that the coefficients of the tap-weight vector really update in all iterations. So, the update rate (UR) of tap-weight vector equation for the SM-IMSAF algorithm will be studied. The update rate is defined as follows

$$
\mathrm{UR}=\frac{N_{\text {update }}}{N_{\text {total }}}
$$

where $N_{\text {update }}$ denotes the real update numbers, $N_{\text {total }}$ represents the whole iterations. 
Table 1. The Case That $\gamma$ Takes Different Values as well as $N=2, P=4$.

\begin{tabular}{|c|c|c|c|c|c|}
\hline$\gamma(i)$ & $\gamma(5)$ & $\gamma(3)$ & $\gamma(1)$ & $\gamma(0.3)$ & $\gamma(0.1)$ \\
\hline $\mathrm{UR}$ & 0.450 & 0.567 & 0.826 & 0.950 & 0.986 \\
\hline
\end{tabular}

Table 2. The Case That $\gamma, P$ Keep the Unchanged Value $\gamma(5), P=4, N$ Takes Different Values

\begin{tabular}{|c|c|c|c|}
\hline$N$ & 2 & 4 & 8 \\
\hline UR & 0.451 & 0.419 & 0.376 \\
\hline
\end{tabular}

Table 3. The Case That $\gamma, N$ Keep the Unchanged Value $\gamma(1), N=2, P$ Takes Different Values

\begin{tabular}{|c|c|c|c|}
\hline$P$ & 2 & 4 & 6 \\
\hline UR & 0.866 & 0.825 & 0.831 \\
\hline
\end{tabular}

From the comparison result (Table 1) above, we find that the update rate will become larger with $\gamma$ takes a smaller value. Moreover, the update rate approximately equals to 1 when $\gamma$ takes $\gamma(0.1)$.

The comparison result (Table 2) shows that there is an inverse relationship between $N$ and the update rate.

From Table 3, the update rate that $P=2$ is larger when comparing with that $P=4$. However, the update rate that $P=6$ is smaller than that $P=4$. To sum up, the value of $\gamma$ has a great impact on the update rate

\section{Conclusions}

In this paper, we have proposed a new algorithm which incorporates the idea of set-membership at the basis of the IMSAF algorithm (SM-IMSAF). The innovation of the proposed SM-IMSAF algorithm lies in the introduction of the set-membership idea. Considering the actual situation, we discuss both the exact-modeling scenario and the under-modeling scenario. The proposed SM-IMSAF algorithm behaves better compared with other relative algorithms, especially in terms of the convergence rate. The simulation results verify the efficiency of the proposed SM-IMSAF algorithm.

\section{Acknowledgment}

This work was partially supported by National Science Foundation of P.R. China (Grant: 61271340, 61571374, and 61433011), and the Fundamental Research Funds for the Central Universities (Grant: SWJTU12CX026).

\section{References}

[1] Kwong, R. H., \& Johnston, E. W. (1992). A variable step size LMS algorithm. IEEE Trans. Signal Processing, 40(7), 1633-1642.

[2] Yu, Y., Zhao, H., \& Chen, B. (2015). Sparseness-controlled proportionate affine projection sign algorithms for acoustic echo cancellation. Circuits Syst. Signal Process, 34(12), 3933-3948.

[3] Lu, L., Zhao, H., He, Z., \& Chen, B. (2015). A novel sign adaptation scheme for convex combination of two adaptive filters. AEU-International Journal of Electronic and Communications, 69(11), 1590-1598.

[4] Lopes, C. G., \& Sayed, A. H. (2007). Incremental adaptive strategies over distributed networks. IEEE Trans. Signal Process, 55(8), 4064-4077.

[5] Shi L., \& Zhao, H. (2016). Variable step-size distributed incremental normalised LMS algorithm. Electronics Letters, 52(7), 519-521.

[6] Haykin, S. (2002). Adaptive Filter Theory (4th ed.). Upper Saddle River, NJ: Prentice-Hall. 
[7] Lee, K. A., \& Gan W. S. (2004). Improving convergence of the NLMS algorithm using constrained Subband Updates. IEEE Signal Processing Letters, 11(9), 736-739.

[8] Abadi, M. S. E., \& Husøy, J. H. (2008). Selective partial update and set-membership subband adaptive filters. Signal Processing, 88(10), 2463-2471.

[9] de Campos, M. L. R., Apolinário, J. A., \& Diniz, P. S. R. (1999). On normalized data-reusing and affine projection algorithms. Proceedings of IEEE Int. Conf. Vol. 2. Electronics, Circuits and Systems (pp. 843-846).

[10] Werner, S., \& Diniz, P. S. R. (2001). Set-membership affine projection algorithm. IEEE Signal Processing Letters, 8(8), 231-235.

[11] Lee, C. W., Lee, Y. K., Chang, M. S., Kim, S. W., \& Park, P. G. (2006). A new affine projection algorithm with data selective method. Proceedings of SICE-ICASE International Joint Conference (pp. 18-21).

[12] Yu, Y., Zhao, H., \& Chen, B. (2015). Sparseness-controlled proportionate affine projection sign algorithms for acoustic echo cancellation. Circuits, Systems, and Signal Process.

[13] Zhao, H., Yu, Y., Gao, S., Zeng, X., \& He, Z. (2014). Memory proportionate APA with individual activation factors for acoustic echo cancellation. IEEE/ACM IEEE Trans. Audio, Speech, Lang. Process, 22(6), 1047-1055.

[14] Zheng, Z., \& Zhao, H. (2015). Memory improved proportionate M-estimate affine projection algorithm. Electronics Letters, 51(6), 525-526.

[15] Yang, F., Wu, M., Ji, P. F., \& Yang, J. (2012). An improved multiband-Structured subband adaptive filter algorithm. IEEE Signal Processing Letters, 19(10), 647-650.

[16] Paleologu, C., Ciochina, S., \& Benesty, J. (2008). Variable step-size NLMS algorithm for under-modeling acoustic echo cancellation. Signal Processing Letters, 15, 5-8.

[17] Abadi, M. S. E., \& Husøy, J. H. (2008). Set-membership subband adaptive filters. Proceedings of ISCCSP, 3rd International Symposium on Communications, Control and Signal Processing (pp. 193-196).

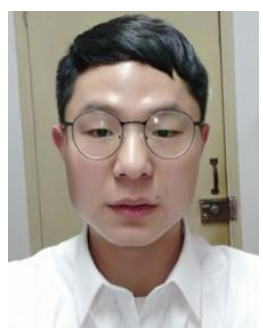

Long Shi was born in Jiangsu Province, China, in 1991. He received the B.E. degree in the School of Electrical Engineering at BinZhou University, Binzhou, China, in 2014. Now he is pursuiting the M.S degree in the field of signal and information processing at the School of Electrical Engineering, Southwest Jiaotong University, Chengdu, China. His current research interest is adaptive signal processing.

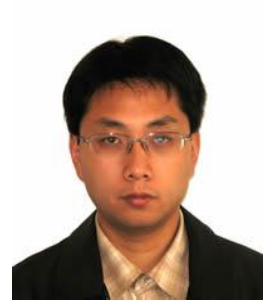

Haiquan Zhao was born in Henan Province, China, in 1974. He received the B.S. degree in applied mathematics in 1998, the M.S. degree and the Ph.D degree in signal and information processing all at Southwest Jiaotong University, Chengdu, China, in 2005 and 2011, respectively. Since August 2012, he was a Professor with the School of Electrical Engineering, Southwest Jiaotong University, Chengdu, China. His current research interests include adaptive filtering algorithm, adaptive Volterra filter, nonlinear active noise control, nonlinear system identification and chaotic signal processing. At present, he is the author or coauthor of more than 50 journal papers and the owner of six invention patents. Prof. Zhao is a member of the IEEE Computational Intelligence Society. He has served as an active reviewer for several IEEE Ransactions, the Institution of Engineering and Technology, and other international journals. 